\title{
Neurosarcoidosis Presenting with Isolated Oculomotor Nerve Palsy
}

\author{
Hye-Rim Shin, Il-Yeon Choo, Eun Young Kim, Yong Woo Shin, Hyung-Min Kwon \\ Department of Neurology, SMG-SNU Boramae Medical Center, Seoul National University College of Medicine, Seoul, Korea
}

Sarcoidosis is a multisystem granulomatous disease. Neurosarcoidosis occurs in approximately $5-15 \%$ of patients with systemic sarcoidosis. Since neurosarcoidosis can manifest in different fashions, diagnosis is sometimes difficult. Here, we present a case of 75-year-old woman with oculomotor neuropathy involving the pupil who had a history of systemic sarcoidosis. We excluded compressive causes of oculomotor neuropathy, such as aneurysm of the posterior communicating artery. Brain magnetic resonance imaging showed enhancement and thickening of the right oculomotor nerve, implying inflammation. Considering the potential for neurosarcoidosis in right oculomotor nerve, we administered high-dose corticosteroid therapy and the patient's clinical symptom and neuroimaging finding improved. Isolated oculomotor nerve palsy in neurosarcoidosis is rare, and this is the first documented case of oculomotor nerve palsy involving the pupil in Korea. We suggest that it is important to recognize inflammatory causes of oculomotor nerve palsy, such as neurosarcoidosis, even in cases that involve the pupil.

J Neurosonol Neuroimag 201\&;10(2):15 $\$-161$

Key Words: Sarcoidosis; Cranial nerve diseases; Oculomotor nerve diseases
Received: July 11, 2018

Revised: August 22, 2018

Accepted: August 28, 2018

Address for correspondence: Hyung-Min Kwon

Department of Neurology, SMG-SNU Boramae Medical Center, Seoul National University College of Medicine, 20 Boramae-ro 5-gil, Dongjak-gu, Seoul o7061, Korea

Tel: +82-2-870-2475

Fax: +82-2-831-2826

E-mail: hmkwon@snu.ac.kr
Sarcoidosis is a multiorgan granulomatous disease of unknown cause. It can involve various organs, such as the lungs, eyes, skin, and lymph nodes, and the nervous system. ${ }^{1}$ Neurosarcoidosis presents in approximately $5-15 \%$ of patients with sarcoidosis, ${ }^{2,3}$ and cranial neuropathy is the most common neurological complication of sarcoidosis. ${ }^{4}$ Facial nerve palsy is the most common cranial neuropathy in neurosarcoidosis, ${ }^{4}$ and optic nerve, vestibulocochlear nerve, and trigeminal nerve involvement have also been reported. ${ }^{3}$

Neurosarcoidosis can manifest in different fashions and reaching a diagnosis may be complicated especially if it presents with rare clinical symptoms. ${ }^{2,5}$ Here, we present a case of neurosarcoidosis that presented with isolated oculomotor nerve palsy.

\section{CASE REPORT}

A 75-year-old woman presented with right eye ptosis and diplopia, which had progressed over 2 months. In her past medical history, she had been admitted to the rheumatology department of our hospital in October 2016 due to a subcutaneous nodule around the left medial malleolus. She also had the respiratory symptom of dyspnea, and fatigue. On the chest X-ray, a pulmonary nodule was seen in right upper lobe. She underwent biopsy of the subcutaneous nodule during the admission, which found a granuloma with multinucleated giant cells. Systemic sarcoidosis was suspected; therefore, she was prescribed $2 \mathrm{mg}$ methylprednisolone and $300 \mathrm{mg}$ hydroxychloroquine daily. She also had a past medical history of hypertension, osteoporosis, idiopathic thrombocytopenic purpura, and Ramsay-Hunt syndrome 6 years ago. She had left peripheral type facial palsy and hypesthesia in the left chin ( $\mathrm{V}_{3}$ area) as sequelae of Ramsay-Hunt syndrome from 6 years ago. She did not have any history of diabetes mellitus, tuberculosis, stroke, or head trauma.

On neurological examination, the pupil of her right 
eye was dilated to $4 \mathrm{~mm}$, and both direct and indirect light reflexes were absent in the right eye. She had limited supraduction, infraduction, and adduction of the right eye, and right upper lid ptosis (Fig. 1A). The Bielschowsky head tilt test result was negative. Left peripheral-type facial palsy and hypesthesia of the left chin were found, sequelae of varicella zoster that had not changed recently. She did not have any pain or paresthesia in her face. Motor power and sensation in all extremities were normal, and both deep tendon reflexes were symmetric and normo-reflexive.

Clinically, isolated right oculomotor nerve palsy was suggested. In routine blood tests, there were no abnormalities other than mild thrombocytopenia, and serology test results for syphilis and human immunodeficiency virus were negative. Test results for vasculitis antibodies, including anti-double strand DNA antibody, antiphospholipid antibody, and antineutrophil cytoplasmic antibody were all negative, and the anticholinesterase test result was negative. Angiotensin converting enzyme was in the normal range (15.9 U/L). Chest X-ray showed a nodular lesion in the right upper lobe without change, which was found to be a fibronodular lesion on chest computed tomography (CT). The sputum acid-fast bacillus smear and culture were both negative.

Brain magnetic resonance imaging (MRI) and angiography (MRA) showed a small vascular outpouching le- sion in the right posterior communicating artery (PcoA) orifice (Fig. 2D). Immediate conventional angiography was performed to rule out PcoA aneurysm, which revealed the outpouching to be the infundibulum. We then performed a further brain MRI with thin sections to assess the cranial nerves more accurately. It showed an ill-defined enhancing lesion in the right cavernous sinus (Fig. 2A) and right oculomotor nerve enhancement with thickening and high signal intensity in the T2-weighted image along the cisternal to extracranial segment (Fig. 2B, C).

On examination of the cerebrospinal fluid (CSF), the opening pressure was $6 \mathrm{~cm} \mathrm{H}_{2} \mathrm{O}$, red blood cells were $\mathrm{o} / \mathrm{mm}^{3}$, and white blood cells were $2 / \mathrm{mm}^{3}$. CSF protein and glucose levels were within the normal range. The CSF gram stain and culture, acid-fast bacillus smear, and polymerase chain reaction of tuberculosis results were all negative, and there were no atypical or malignant cells on cytology.

Considering the past history of systemic sarcoidosis, we suspected neurosarcoidosis presenting with isolated right oculomotor nerve palsy. Consequently, we started high-dose intravenous methylprednisolone (1 g daily) treatment for five days, followed by transition to oral prednisolone (6o mg daily). Oral prednisolone was tapered out by $10 \mathrm{mg}$ every week. Five days after the treatment, ptosis of the right upper lid and diplopia slowly improved. Repeated brain MRI with the cranial nerve
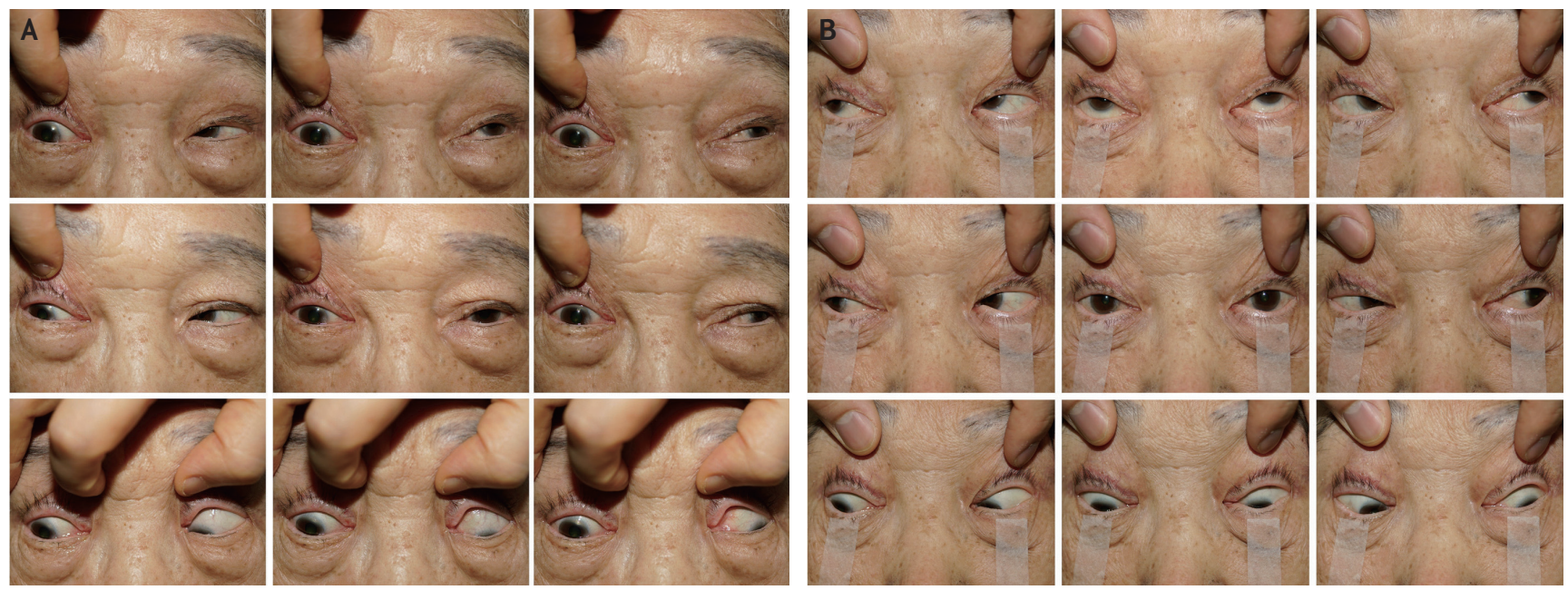

FIG. 1. Nine-gaze photographs before and after corticosteroid therapy. (A) Nine-gaze photographs on the first day of admission. It shows exotropia of the right eye in the primary position, and limitation of adduction, supraduction, and infraduction, which is consistent with right oculomotor nerve palsy. (B) Nine-gaze photograph 10 days after discharge. Compared to the initial nine-gaze photograph, it shows recovery from exotropia of the right eye in the primary position and normalized extraocular movement of the right eye. 
protocol nine days after treatment showed a decreased size of the enhancing lesion in the right cavernous sinus (Fig. 2E). Enhancement with thickening, and high signal intensity on T2-weighted images of the right oculomotor nerve were also improved (Fig. 2F, G).

She was discharged with oral prednisolone and hydroxychloroquine. A nine-gaze photo was taken 10 days after discharge and showed no limitation of eye movement (Fig. 1B). The size of the pupil of right eye decreased to $3 \mathrm{~mm}$, and the light reflexes of the right eye also recovered. The patient is now maintained on oral prednisolone (12.5 mg), hydroxychloroquine (300 $\mathrm{mg}$ once daily), and mycophenolate sodium (360 mg twice daily) in the rheumatology clinic. Neither recurrence nor aggravation of the oculomotor nerve palsy had reoccurred up to now.

\section{DISCUSSION}

Sarcoidosis is a multisystem disease that has variety of clinical presentations including neurosarcoidosis. ${ }^{2,3}$ Neurosarcoidosis may difficult to diagnose since it has variable clinical presentation. ${ }^{2,5}$

Cranial neuropathy is most common presentation of neurosarcoidosis. ${ }^{2,4}$ However, oculomotor nerve palsy is rare and usually occurs with other cranial neuropathies in the context of multiple cranial neuropathies. ${ }^{6}$ In a case series of 68 neurosarcoidosis patients who were followed up, 2.5\% of 68 patients with neurosarcoidosis had oculomotor nerve palsy as one of the neurological symptoms. ${ }^{7}$ In another retrospective study, oculomotor nerve dysfunction occurred in only two of 33 patients with neurosarcoidosis. ${ }^{4}$ Isolated oculomotor nerve palsy is rarely reported ${ }^{8,9}$ and cases involving the pupil are very rare. ${ }^{9}$

In oculomotor nerve palsy involving the pupil, immediate brain imaging, including MRA or CT angiography, should be performed to allow differentiation from compressive lesions such as PcoA aneurysm. In this case, we excluded the possibility of a compressive lesion (i.e., Pcom aneurysm) by thorough work-ups
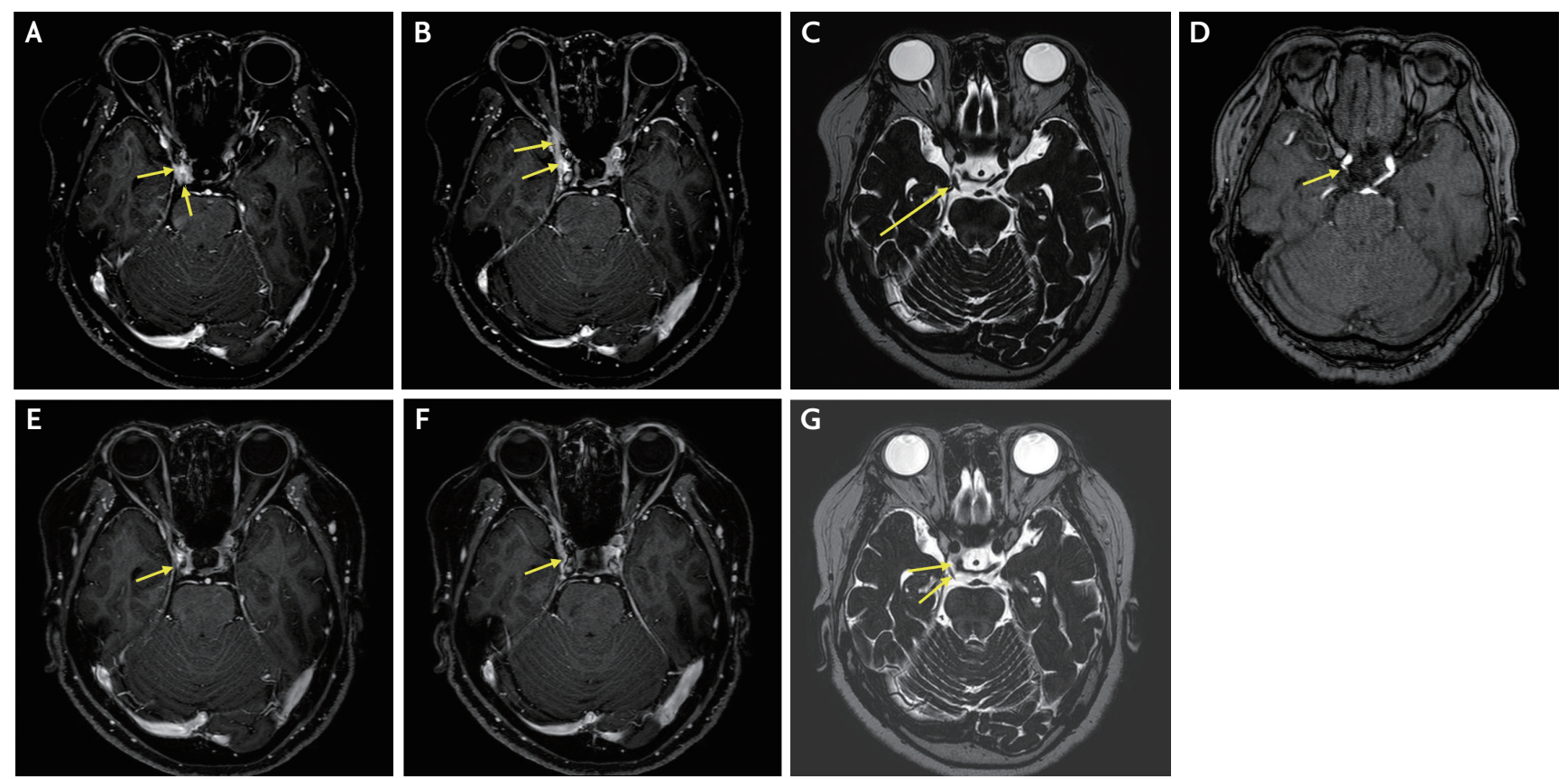

FIG. 2. Cranial nerve brain MRI before and after the corticosteroid therapy. (A-D) Initial brain MRI and MRA of the patient. It shows an ill-defined enhancing lesion (arrows) in the right cavernous sinus (A). The right oculomotor nerve (arrows) shows enhancement with thickening on a gadolinium-enhanced T1-weighted image (B), and mild hyperintense changes on the T2-weighted image (C). On MRA, a small vascular outpouching lesion (arrow) is seen in the right posterior communicating artery orifice. It was revealed to be the infundibulum on conventional angiography (D). (E-G) Brain MRI 9 days after therapy with high-dose methylprednisolone. Compared to the initial brain MRI, it shows decreased size of the enhancing lesion (arrow) in the right cavernous sinus (E), improved enhancement (arrow) with thickening on the gadolinium-enhanced T1-weighted image (F), and hyperintense change on the T2-weighted image (G) of the right oculomotor nerve (arrows). MRI; magnetic resonance imaging, MRA; magnetic resonance angiography. 
including brain MRI, MRA, and conventional angiography. Inflammatory diseases such as neurosarcoidosis are known to respond well to corticosteroid therapy. ${ }^{2,5}$ Therefore, when a compressive lesion is excluded, we should consider an inflammatory etiology for appropriate management.

To diagnose sarcoidosis, the chest X-ray evidence should be accompanied by compatible clinical features, with non-caseating granuloma on biopsy. ${ }^{1,10}$ In sarcoidosis, the chest X-ray commonly shows bilateral hilar lymphadenopathy, but infiltration alone or fibrotic bands without hilar lymphadenopathy can also be seen. ${ }^{10}$ In this case, systemic sarcoidosis can be diagnosed, since the patient had symptoms of fatigue and dyspnea with a fibronodule on chest X-ray. Furthermore, the biopsy of a skin nodule in 2016 found a non-caseating granuloma.

To the best of our knowledge, this is the first case of neurosarcoidosis presenting with oculomotor nerve palsy with pupil involvement without any other neurologic symptoms in Korea. We diagnosed a rare case of neurosarcoidosis after excluding compressive lesions with a thorough work-up, and the patient was treated successfully with high-dose corticosteroid therapy.

\section{Conflicts of Interest}

No potential conflicts of interest relevant to this article was reported.

\section{REFERENCES}

1. Sharma OP. Definition and history of sarcoidosis. In: Sarcoidosis. Drent M, Costabel U. European Respiratory Monograph. Vol. 10. Sarcoidosis: ERS Journals Ltd., 2005;112.

2. Hoitsma E, Faber CG, Drent M, Sharma OP. Neurosarcoidosis: a clinical dilemma. Lancet Neurol. 2004;3:397-407.

3. Tavee JO, Stern BJ. Neurosarcoidosis. Continuum (Minneap Minn). 2014;20 (3 Neurology of Systemic Disease):545-559.

4. Stern BJ, Krumholz A, Johns C, Scott P, Nissim J. Sarcoidosis and its neurological manifestations. Arch Neurol. 1985;42:909-917.

5. Ibitoye RT, Wilkins A, Scolding NJ. Neurosarcoidosis: a clinical approach to diagnosis and management. J Neurol. 2017;264:1023-1028.

6. Velazquez A, Okun MS, Bhatti MT. Bilateral third nerve palsy as the presenting sign of systemic sarcoidosis. Can J Ophthalmol. 2001;36:416-419.

7. Zajicek JP, Scolding NJ, Foster O, Rovaris M, Evanson J, Moseley IF, et al. Central nervous system sarcoidosis--diagnosis and management. QJM. 1999;92:103-117.

8. Ueyama H, Kumamoto T, Fukuda S, Fujimoto S, Sannomiya $\mathrm{K}$, Tsuda T. Isolated third nerve palsy due to sarcoidosis. Sarcoidosis Vasc Diffuse Lung Dis. 1997;14:169-170.

9. Bansal S, Yin K, Vishwanath M, Doran H, Laitt R, Ansons A. Isolated pupil-involving third nerve palsy as the first presentation of sarcoidosis. Neuroophthalmology. 2014;38:278-28o.

10. Iannuzzi MC, Rybicki BA, Teirstein AS. Sarcoidosis. N Engl J Med. 2007;357:2153-2165. 